\title{
DEVELOPMENT OF A NOVEL HYBRID TYPE MAGNETIC BEARING AND APPLICATION TO SMALL IMPELLER CENTRIFUGAL PUMP FOR ARTIFICIAL HEARTS
}

\author{
Takashi Saito, Toru Masuzawa, and Naohisa Nakayama
}

\begin{abstract}
We have developed a new hybrid magnetic (HM) bearing system for durable and small size artificial hearts. A prototype maglev pump was also developed to verify the feasibility of the developed HM bearing system. A small impeller centrifugal pump was combined with the HM bearing for the prototype pump. The impeller is levitated by a set of HM bearings at both sides of the impeller. A motor stator is set at the center of the device to rotate the levitated impeller. Maximum rotating speed with complete levitation in the air is $15,000 \mathrm{rpm}$. Maximum oscillation amplitude in the radial direction is $0.04 \mathrm{~mm}$ with a target rotating number of 5,000 rpm. The developed HM bearing system indicated sufficient levitation performance for the artificial heart and the magnetically levitated centrifugal pump with small impeller also displayed good pump performance as a ventricular assist system.
\end{abstract}

\section{Key Words}

Artificial heart, maglev technology, hybrid type magnetic bearing, centrifugal pump

\section{INTRODUCTION}

Durability of the mechanical bearing and seal determin the lifetime of the artificial heart pump. Several groups in the world are trying to develop a magnetically levitated pump to eliminate these parts [1]-[3]. We have also developed several types of magnetically levitated centrifugal pumps [4]-[7]. The size of the device should be small enough for the artificial heart. Therefore, we

received 18th November 2005,

final received 1st August 2006.

Contact to: Toru Masuzawa, Dr. Eng.,

Dept. of Mechanical Engineering, Ibaraki University,

4-12-1 Nakanarusawa, Hitachi, Ibaraki, 316-8511 Japan.

email:masuzawa@mx.ibaraki.ac.jp, have developed a compact maglev system by using a hybrid magnetic (HM) bearing for a small pump for artificial hearts. Also, we are trying to develop a smaller size centrifugal pump to reduce the device size. In this paper, the structure of the newly developed HM bearing and the centrifugal pump with a smaller impeller will be reported.

\section{METHOD}

\section{HYBRID MAGNETIC BEARING}

Figure 1 shows the schematic diagram of a HM bearing. This bearing has four core pieces and four permanent magnets to produce bias magnetic flux. A core piece has a main pole and two sub poles. The main pole has a wound 
coil for the electromagnet. Bias permanent magnets are set between the core pieces. The magnetic circuit of this bearing is constructed on the two dimensional plane. Therefore, this new HM bearing has relatively higher efficient performance than conventional HM bearing from the point of view smaller leakage flux.

The magnetic flux pass way in the HM bearing is shown in figure 2. The dashed line indicates the bias magnetic flux produced by the permanent magnets. The bias magnetic flux passes through the core pieces and the rotor. The full line indicates the control magnetic flux generated by the electromagnets. The control magnetic flux passes through the main pole, the radial air gap of the rotor, the magnetic core of the rotor, and the sub poles. The radial position of the rotor is controlled by regulating each magnetic flux of the electromagnets. Two opposite electromagnets are used in a push pull manner to control the radial position. In figure 2 , magnetic flux in the radial air gap of the main pole of the upper side core is increased with the electromagnet flux, but that of bottom side pole is decreased.

The magnetic circuit of the HM bearing is modeled by using the analogy of the electric circuit. An electric equivalent circuit of the HM bearing is shown in figure 3 . The magnetic resistance of the air gap at the main pole is represented as resistance $R$. The resistance of the air gap at the sub pole is coefficient $k$ times resistance $R$ because the gap length at the sub pole is different with that at the main pole. The cross'sectional area of the sub pole is set half of that of the main pole. The magnetic resistance of the permanent magnet is assumed as infinity. $\mathrm{Vp}$ and Ve represent the magnetomotive forces of the permanent magnet and the electric magnet. In figure 3 , Ve is set at the upper and bottom cores to model the push pull bearing. When the magnetic resistance is not changed, attractive force $\mathrm{F}$ is represented as follows.

$$
F=\frac{k-4}{(k+2) k \mu_{0} A} V_{m} V_{e}
$$

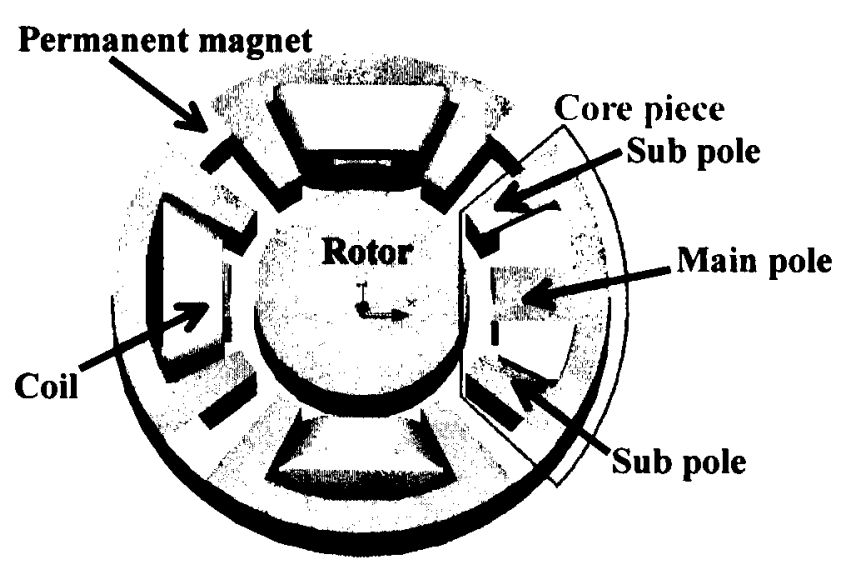

Fig.1 Novel hybrid type magnetic bearing

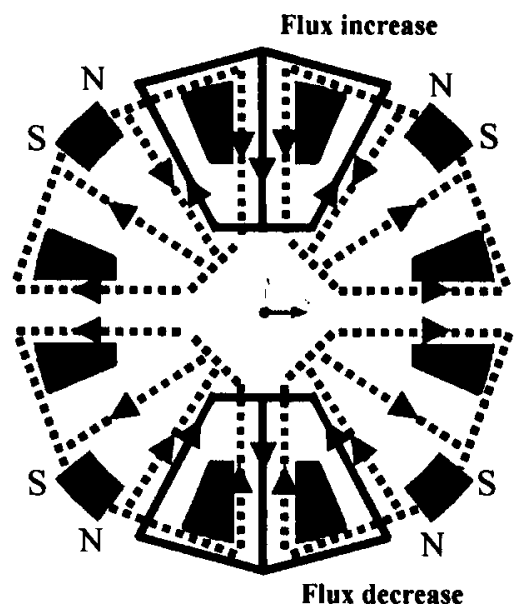

Fig.2 Magnetic flux pass way for radial position control

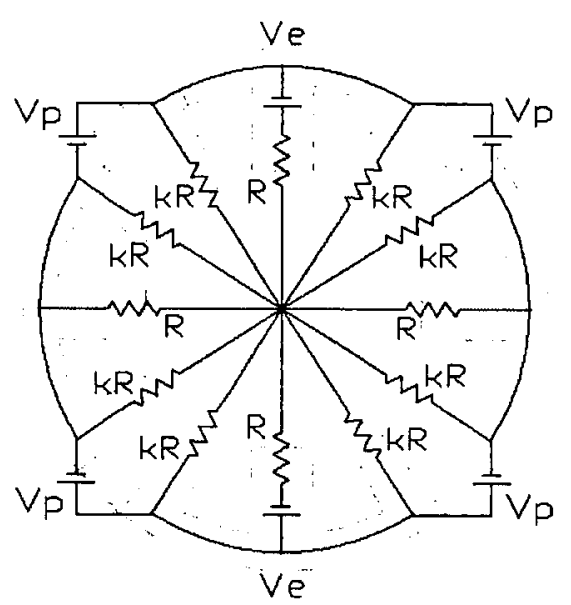

Fig.3 Electric equivalent circuit of the Magnetic circuit 
where, $\mu_{0}$ is vacuum permeability, A is cross-sectional area of the mail pole. Therefore, The attraction can be controlled by using the magnetomotive force of the electric magnet coil that be regulated by changing the current through the electric coil.

Two dimensional magnetic field analysis based on the finite element method was performed to determine suitable design parameter's value of the HM bearing. ANSYS, a commercial simulation program, was used for the analysis. Design criteria of the HM bearing are following two criteria. One of the criteria is to prevent the magnetic flux saturation. Another criterion is to produce sufficient radial force. Figure 4 shows the magnetic flux intensity in the HM bearing, that is one of the results of the two dimensional magnetic field analysis. In this analysis, the air gap between the core pole edge and the levitated rotor is set to $1 \mathrm{~mm}$ to make a space for the casing wall and the blood gap. The diameter of the HM bearing is set to $60 \mathrm{~mm}$. Turn number of the each electro- magnet became 200 turns.

\section{PROTOTYPE OF MAGLEV CENTRIFUGAL PUMP WITH SMALLER IMPELLER}

The schematic diagram and exterior view of the prototype maglev centrifugal pump are shown in Figures 5 and 6 . The prototype pump was developed to verify the feasibility of the HM bearing. Therefore, the prototype system is a basic structure with two radial HM bearings and a motor mechanism. The diameter of the smaller impeller is $30 \mathrm{~mm}$. The diameter size was determined for developing a smaller size device in the future. HM bearings are set at both edges of the rotor impeller. A motor stator is set at the center part of the pump. The impeller part has a shaft, which has a channel hole at the center. A ferrite permanent magnet with four poles for rotation control is set on the center of the shaft surface. The rotor core is also constructed at both edges of the shaft to attach the electromagnetic soft iron. The vanes are constructed at the edge of the impeller shaft. The blood will pump through the inlet port, the channel hole,

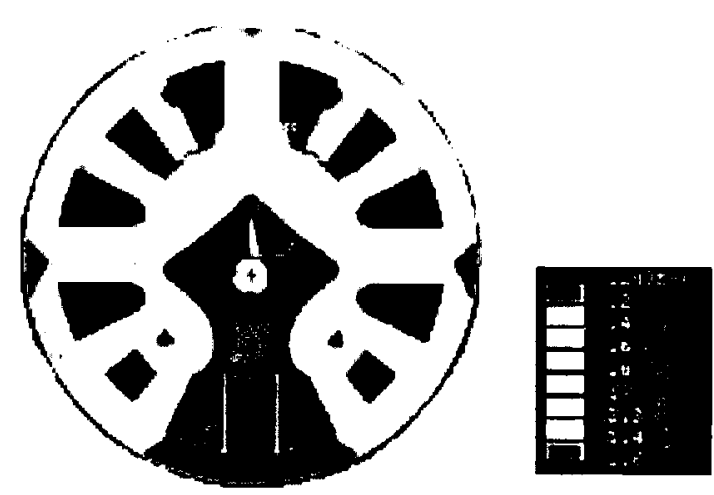

Fig.4 Results of the two dimensional magnetic field analysis

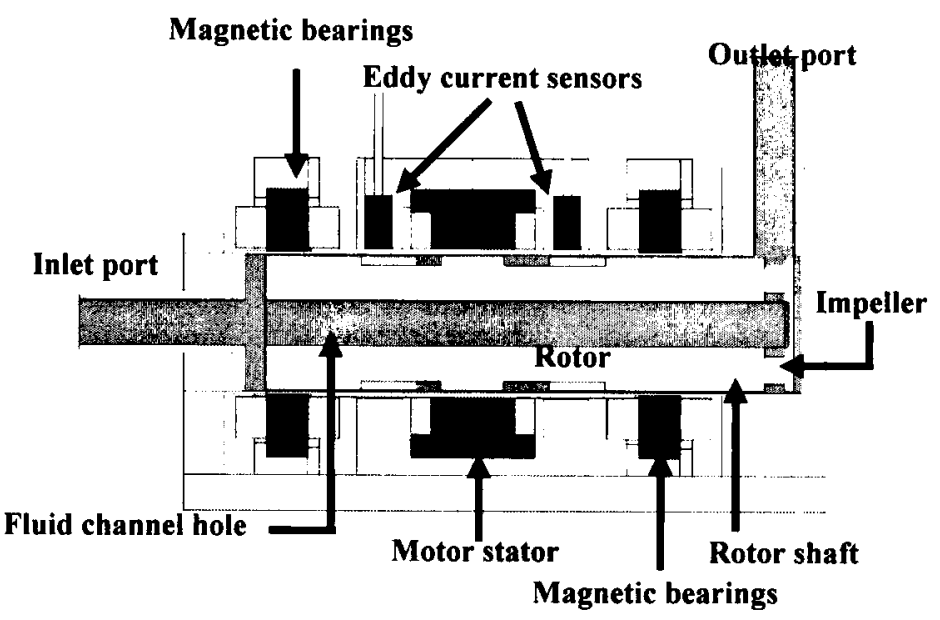

Fig.5 Schematic diagram of the prototype maglev pump

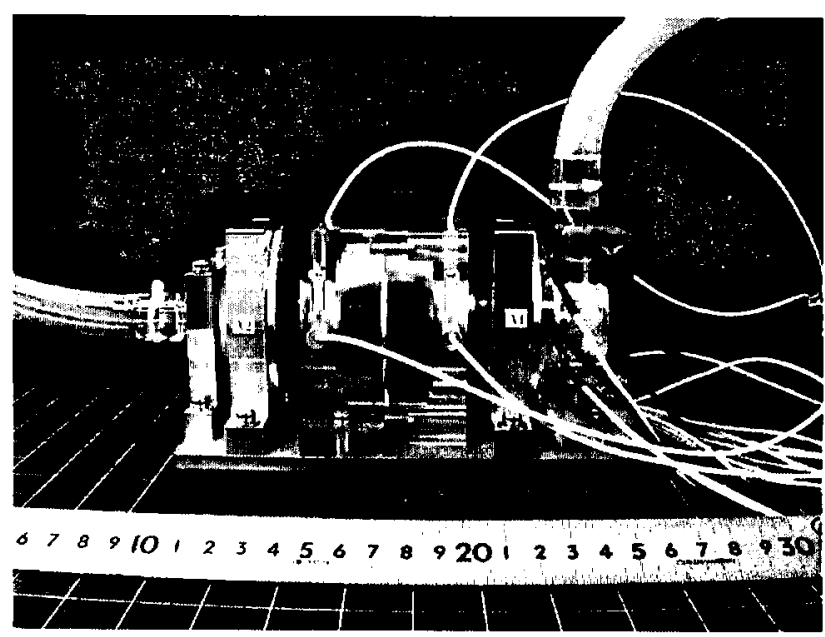

Fig.6 Exterior view of the prototype maglev pump 
the impeller vanes, and the outlet port. The total length, height and width of the prototype pump are $180 \mathrm{~mm}, 112$ $\mathrm{mm}$ and $60 \mathrm{~mm}$, respectively.

\section{LEVITATION CONTROL SYSTEM}

Figure 7 shows the schematic diagram of the levitation control system. The rotor levitation and rotation are controlled by a digital PID control algorithm using the DSP system (dSPACE :DS1104). The rotor positions in the radial direction are measured by using four eddy current sensors and the control currents to the HM bearing are calculated by the DSP system. Control current signals are transfered via a $\mathrm{D} / \mathrm{A}$ converter to the power amplifier to provide currents to the electromagnets of the HM bearings and the motor.

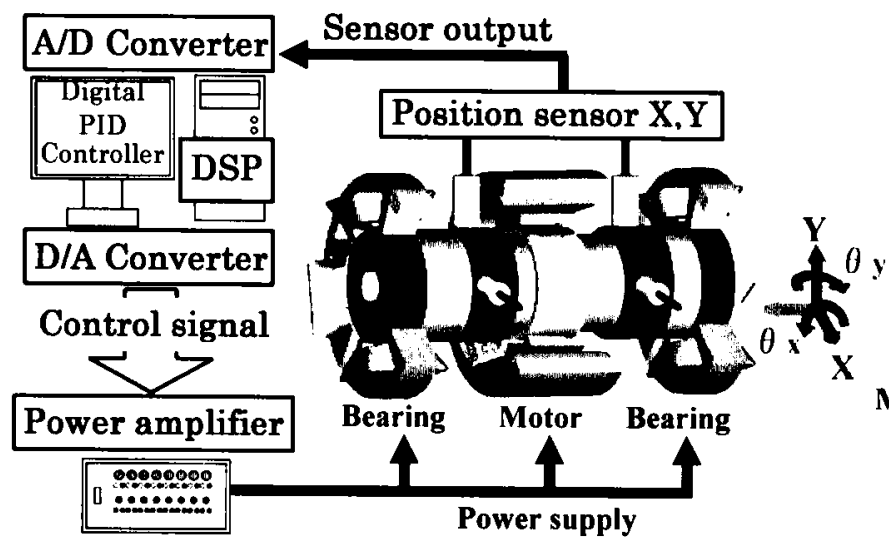

Fig.7 Schematic diagram of the levitation control system

\section{EXPERIMENTS}

At first, the produced radial force of the HM bearing was measured by constructing a special measurement setup shown in figure 8 . A $300 \mathrm{~mm}$ length shaft is attached to the rotor core to measure the attractive force. The rotor core attached to the long shaft is supported by using the developed HM bearing. Another side of the long shaft is supported by a mechanical ball bearing. Therefore, the rotor core could be moved in the radial direction freely and is restricted only in the axial direction. The load transducer is connected to the end of the shaft and the attractive force during the position control of the rotor core is measured directly.
The levitation performance of the maglev system during rotation was also evaluated with the prototype maglev pump system. The oscillation amplitude of the levitated impeller during the rotation in the air was measured by using four eddy current sensors. Finally, the pump performance of the miniaturized maglev centrifugal pump was evaluated with a closed mock circuit filled with water. Head pressure was calculated by using the signals from the pressure sensors set on the inlet and the outlet port. Flow rate at the outlet port was measured by using an electromagnet flow meter.

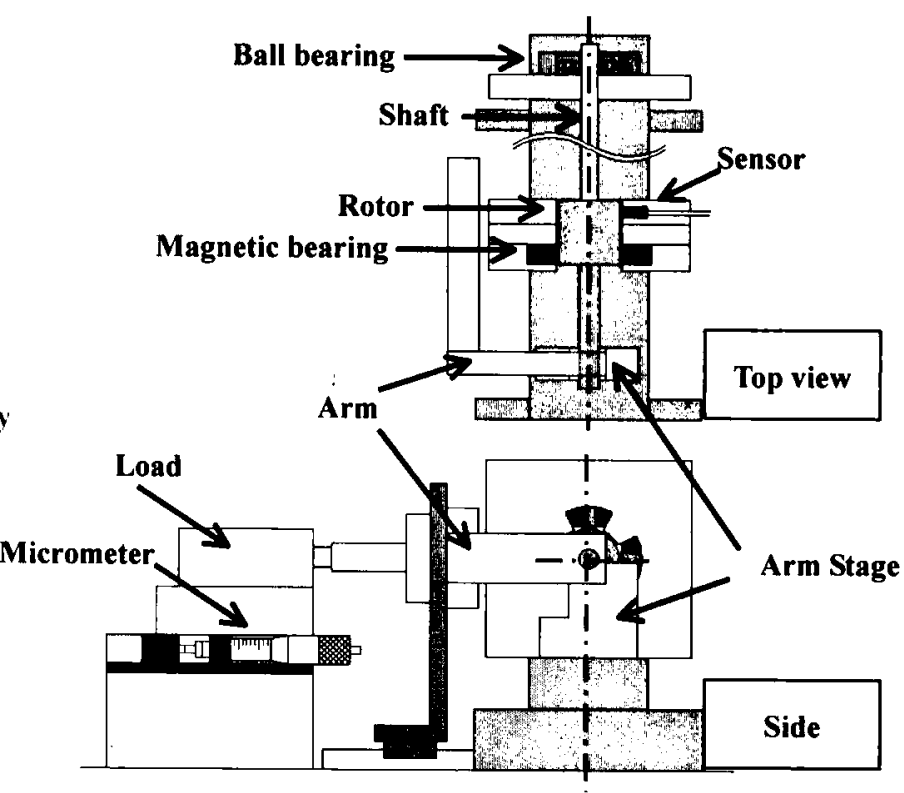

Fig.8 Experimental setup to measure the produced force of the $\mathrm{HM}$ bearing

\section{RESULTS}

Figure 9 shows the relationship between the electromagnet current and the produced force in the radial direction. The rotor is kept at the center of the bearing in this experiment. Maximum produced force was more than $8.5 \mathrm{~N}$.

Figure 10 shows the levitation performance of the developed HM bearing during rotation. Maximum rotating speed with complete levitation in the air was $15,000 \mathrm{rpm}$. The oscillation amplitude in the radial direction was $0.04 \mathrm{~mm}$ under a target rotation number of 


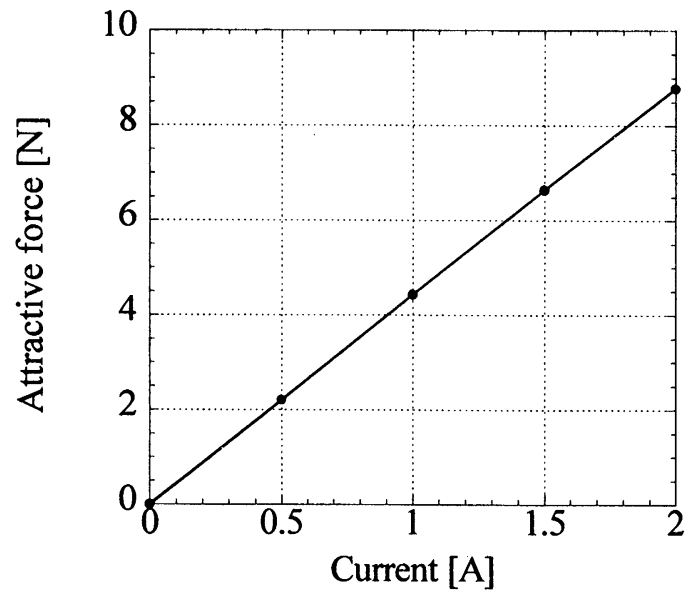

Fig.9 Relationship between drive current and produced force of the HM bearing

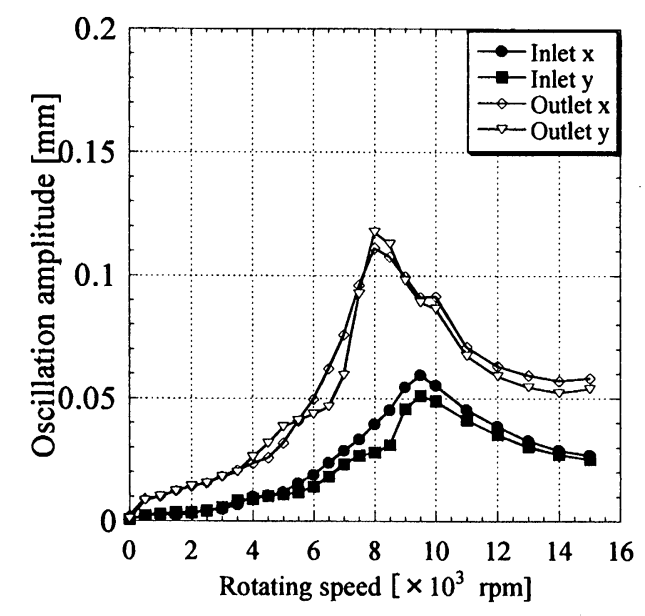

Fig.10 Relationship between maximum oscillation amplitude and rotating speed of the levitated impeller

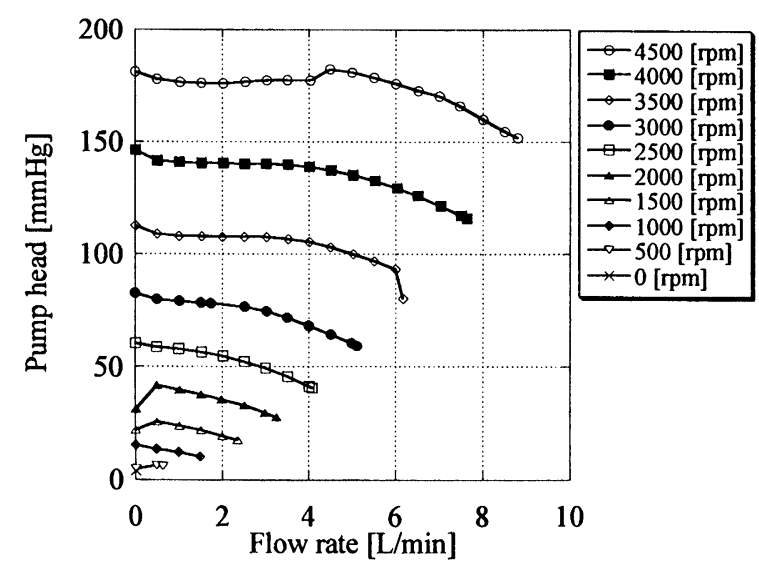

Fig.11 Head pressure - flow rate characteristics of the prototype maglev pump

\section{5,000 rpm.}

Figure 11 shows head pressure-flow rate (HQ) characteristics of the developed pump. The maximum head pressure and the maximum flow rate were $180 \mathrm{~mm}$ $\mathrm{Hg}$ and $8.8 \mathrm{~L} / \mathrm{min}$, respectively.

\section{DISCUSSION}

Basically, the three dimensional magnetic pass way is constructed in the HM bearing to avoid the interference between the magnetic flux produced by the electric magnet and the permanent magnet. A complex magnetic pass way is the cause of magnetic flux leakage and bigger device size [8]. Therefore, we designed a new HM bearing which is smaller size with two dimensional pass way to achieve less magnetic flux leakage. The developed HM bearing displayed sufficient performance for artificial hearts. However, there is still leakage magnetic flux between the sub poles because the distance between poles is too short in the small bearing. Optimization of the core shape will be needed as a next study step.

One of the targets of the HM bearing for the artificial heart is sufficient attractive force to overcome artifacts. Current bearings can produce an attractive force more than $8 \mathrm{~N}$ with the 200 grams rotor. This means that the developed bearing can support the levitated impeller against an acceleration of $4 \mathrm{G}$. The acceleration when running is $1.3 \mathrm{G}$ and the developed bearing can support the levitated impeller in such a condition.

The levitated rotor could be rotated up to a rotating number of $15,000 \mathrm{rpm}$ in the air and up to $4,500 \mathrm{rpm}$ in the water with the prototype pump. We suppose that the small centrifugal pump will usually be driven in the rotation number range from $1,000 \mathrm{rpm}$ to $5,000 \mathrm{rpm}$. The prototype system could drive the levitated impeller in the target rotational number range and the feasibility of the developed HM bearing system was confirmed.

Our target pump performance for the assist device has a 
head pressure of $200 \mathrm{~mm} \mathrm{Hg}$ and a flow rate of $10 \mathrm{~L} / \mathrm{min}$. The developed pump indicated good pump performance. The limitation of the pump performance was determined by the magnetic suspension limitation. The axial position of the levitated impeller was shifted with a thrust fluid force in the water, because the axial position of the rotor impeller is not controlled actively in the system. As a result, the levitated impeller touched the casing at a rotating number of $5,000 \mathrm{rpm}$. The axial position compensator of the rotor impeller against the thrust fluid force should be added to achieve better pump performance with higher rotation speed. However, it was clarified that the smaller size impeller with a $30 \mathrm{~mm}$ diameter could produce enough pump performance as a ventricular assist device.

Power consumption and efficiency of the device is important for the development of the artificial heart. Input power into the HM bearing was 4 watts during the rotation with a rotating number of $2500 \mathrm{rpm}$. A feasibility test is focused on in this study. Then the magnetic materials, that minimize the power consumption, are not used. The power consumption can be reduced by using better materials to prevent eddy current in the magnet core. Also, an inexpensive ferrite permanent magnet was used for the motor in the study. The neodymium magnet should be used to achieve higher efficiency.

The pump size could be reduced by changing the structure of the magnetic system in the future. The length of the device will be decreased to about $70 \mathrm{~mm}$ by adopting a self-bearing motor. We have already finished designing the self-bearing motor for this device and will report about the miniaturization of the pump in the near future.

\section{CONCLUSION}

A novel hybrid magnetic bearing for a smaller size artificial heart has been invented and applied to construct a magnetically suspended centrifugal pump with a small impeller. The developed HM bearing indicated sufficient levitation performance. The prototype maglev pump also displayed good pump performance as a ventricular assist system and the feasibility of the new HM bearing was confirmed. A smaller magnetically suspended artificial heart will be constructed with the newly developed HM bearing in the future.

\section{ACKNOWLEDGEMENT}

This work was supported financially in part by a Grant-in-Aid for Scientific Research (B) from Japan Society for the Promotion of Science (no.14380389).

\section{REFERENCES}

1. Schoeb R, Barletta N, Fleischli A, Foiera G, Gempp T, Reiter H-G, Poirier VL, Gernes DB, Bourque K, Loree HM, Richardson JS: A bearingless motor for a left ventricular assist device (LVAD). 7th ISMB August 23-25: 383-388, 2000.

2. Nojiri C, Kijima T, Maekawa J, Horiuchi K, Kido T, Sugiyama T, Mori T, Sugiura N, Asada T, Shimane H, Nishimura K, Ban T, Akamatsu T, Ozaki T, Ito H, Suzuki M, Akutsu T: More than 1 year continuous operation of a centrifugal pump with a magnetically suspended impeller. ASAIO Journal 43-5: 548-552, 1997.

3. Allaire P, Hilton E, Baloh M, Maslen E, Bearson G, Noh D, Khanwilkar P, Olsen D: Performance of a continuous flow ventricular assist device; magnetic bearing design, construction, and testing. Artificial Organs, 22-6: 475-480, 1998.

4. Masuzawa T, Onuma H, Kim SJ, Okada Y: Magnetically suspended centrifugal blood pump with a self-bearing motor. ASAIO Journal 48: 437-442, 2002.

5. Masuzawa T, Ezoe S, Kato T, Okada Y: Magnetically suspended centrifugal blood pump with an axial levitated motor, Artificial Organs, 27-7: 631-638, 2003.

6. Onuma $\mathrm{H}$, Masuzawa $\mathrm{T}$, Matuda $\mathrm{K}$, Okada $\mathrm{Y}$ : Magnetically levitated centrifugal blood pump with radially suspended self-bearing motor. 8th ISMB August 26-28:3-8, 2002

7. Onuma H, Murakami M, Masuzawa T: Novel Maglev Pump With a Combined Magnetic Bearing. ASAIO Journal 51: 50-55, 2005.

8. Okada Y, Koyanagi H, Kakihara $\mathrm{K}$, and Ueno S: MiracBearing: new Concept of Miracle Magnetic Bearings, Prof. of 9th Int. Symp. on Magnetic Bearings, Lexington, Kentucky, USA, August 3-6, 2004 